PROCEEDINGS OF THE

AMERICAN MATHEMATICAL SOCIETY

Volume 140, Number 4, April 2012, Pages 1437-1443

S 0002-9939(2011)11304-2

Article electronically published on September 1,2011

\title{
ON THE GRADIENT ESTIMATE OF CHENG AND YAU
}

\author{
OVIDIU MUNTEANU
}

(Communicated by Michael Wolf)

\begin{abstract}
We improve the well-known local gradient estimate of Cheng and Yau in the case when the Ricci curvature has a negative lower bound.
\end{abstract}

\section{INTRODUCTION}

Let $M$ be an $m$-dimensional complete noncompact Riemannian manifold. The local Cheng-Yau gradient estimate is a standard result in Riemannian geometry; see [2], also cf. [4]. It asserts that for $f: B_{p}(2 R) \rightarrow \mathbb{R}$ harmonic and positive, if the Ricci curvature on $B_{p}(2 R)$ has a lower bound Ric $\geq-(m-1) K$ for some $K \geq 0$, then

$$
\sup _{B_{p}(R)}|\nabla \log f| \leq(m-1) \sqrt{K}+\frac{C}{R} .
$$

Notice that if $K=0$, it follows that a harmonic function with sublinear growth on a manifold with nonnegative Ricci curvature is constant. This result is clearly sharp since on $\mathbb{R}^{n}$ there exist harmonic functions which are linear.

When $K>0$, we can rescale the metric so that we take $K=1$. In particular, if $f$ is positive harmonic on $M$ and Ric $\geq-(m-1)$ on $M$, then it follows that $|\nabla \log f| \leq m-1$ on $M$. This result is also sharp; in fact, the equality case was recently characterized in 4 .

This means that for $K=1$ in (1) the factor $m-1$ on the right-hand side is sharp. However, the correction term that depends on the radius is not sharp anymore. The purpose of this paper is to prove a sharp version of the local Cheng-Yau gradient estimate in the following form.

Theorem 1. Let $M$ be a complete noncompact Riemannian manifold of dimension $m$ with Ricci curvature bounded from below on a geodesic ball $B_{p}(2 R)$ by

$$
\text { Ric } \geq-(m-1) .
$$

There exist constants $C_{1}$ depending only on $m$ and $C_{2}$ a universal constant such that if $f: B_{p}(2 R) \rightarrow \mathbb{R}$ is a positive harmonic function, then

$$
\sup _{x \in B_{p}(R)}|\nabla \log f|(x) \leq m-1+\frac{C_{1}}{R} \exp \left(-C_{2} R\right) .
$$

Received by the editors December 28, 2010.

2010 Mathematics Subject Classification. Primary 53C21; Secondary 58J05.

The author's research was partially supported by NSF grant No. DMS-1005484.

(C)2011 American Mathematical Society 
Certainly, our estimate becomes meaningful for large $R$. Let us also remark that by rescaling the metric we obtain that if $R i c \geq-(m-1) K$ on $B_{p}(2 R)$ and $f$ is positive harmonic on $B_{p}(2 R)$, then

$$
\sup _{B_{p}(R)}|\nabla \log f| \leq(m-1) \sqrt{K}+\frac{C_{1}}{R} \exp \left(-C_{2} \sqrt{K} R\right) .
$$

The Cheng-Yau gradient estimate is a fundamental tool in geometric analysis. The technique has been applied in various situations; for an overview of the subject see e.g. [5, 3. In many applications the value of the constants in the estimate is quite important. For example, the sharp version of the global estimate in the case when Ric $\geq-(m-1)$ on $M$ has been very instrumental in rigidity theorems; see [4] and 6]. The importance of our theorem, besides being sharp, is that the estimate does not diverge when integrated on minimizing geodesics. For example, in Corollary 1 in this paper we have established a sharp lower bound for the Green's function on a nonparabolic manifold with a negative lower bound for Ricci curvature. Similar sharp upper or lower bound estimates can be established for harmonic functions defined on manifolds with boundary.

\section{Proof of the Result}

Proof. The proof of the theorem follows the standard argument of Cheng and Yau. We apply the Bochner technique to $\phi^{2}|\nabla \log f|^{2}$, where $\phi$ is a cutoff function with support in $B_{p}(R)$. The difference here is that we use a judicious choice of cutoff $\phi$ which improves the argument.

We will prove the following statement: Let $f: B_{p}(2 R) \rightarrow \mathbb{R}$ be positive and harmonic, where $R \geq R_{0}(m)$ with $R_{0}$ depending only on $m$.

Assume that Ric $\geq-(m-1)$ on $B_{p}(2 R)$. Then

$$
|\nabla \log f|(p) \leq m-1+C_{1} \exp \left(-C_{2} R\right) .
$$

It is evident that (2) proves Theorem 1, because we can apply (2) for each $x \in$ $B_{p}(R)$. Of course, since (2) is true for any radius, we can apply it for the radius $\frac{R}{2}$, with $R$ the same as in Theorem 1 .

Let $h=\log f$. Consider also the function $\phi:[0, \infty) \rightarrow \mathbb{R}$ defined by

$$
\begin{aligned}
& \phi(0)=1, \\
& \phi(r)=1-\exp \alpha\left(R-\frac{R^{2}}{r}\right) \text { for } 0<r \leq R, \\
& \phi(r)=0 \text { for } r>R,
\end{aligned}
$$

where $\alpha>0$ is a (small) number that will be set later.

We compute directly that for $r<R$,

$$
\begin{aligned}
\phi^{\prime}(r) & =-\alpha \frac{R^{2}}{r^{2}} \exp \alpha\left(R-\frac{R^{2}}{r}\right), \\
\phi^{\prime \prime}(r) & =\left(2 \alpha \frac{R^{2}}{r^{3}}-\alpha^{2} \frac{R^{4}}{r^{4}}\right) \exp \alpha\left(R-\frac{R^{2}}{r}\right), \\
\phi^{\prime}(0) & =\phi^{\prime \prime}(0)=0 .
\end{aligned}
$$

By applying $\phi$ to the distance function from $p$ we obtain a cutoff function on $M$, with support in $B_{p}(R)$, which we continue to denote with $\phi$, i.e. $\phi(x)=\phi(r(x))$. 
Let us now consider $G: B_{p}(R) \rightarrow \mathbb{R}$,

$$
G=\phi^{2}|\nabla h|^{2} .
$$

Since $G$ is nonnegative on $B_{p}(R)$ and $G=0$ on $\partial B_{p}(R)$, it follows that $G$ attains a maximum point in the interior of $B_{p}(R)$. Let $x_{0}$ be this maximum point. We will assume from now on that $r(x)=d(p, x)$ is smooth at $x_{0}$, so $\phi$ and $G$ are also smooth at $x_{0}$. If this is not the case; then one can use a support function at $x_{0}$, which is smooth. The computations will be similar and will imply the same result. In this standard argument, it is essential that $\phi(r)$ be nonincreasing, which is true in our case; see [1].

Hence at $x_{0}$ we have, by the maximum principle:

$$
\begin{aligned}
\left|\nabla G\left(x_{0}\right)\right| & =0, \\
\Delta G\left(x_{0}\right) & \leq 0 .
\end{aligned}
$$

The following part of the argument is well known, but we include it for completeness. By the Bochner formula, since $h=\log f$ and $\Delta h=-|\nabla h|^{2}$, we get

$$
\begin{aligned}
\frac{1}{2} \Delta|\nabla h|^{2} & =\left|h_{i j}\right|^{2}+\langle\nabla h, \nabla(\Delta h)\rangle+\operatorname{Ric}(\nabla h, \nabla h) \\
& \geq\left|h_{i j}\right|^{2}-\left\langle\nabla h, \nabla|\nabla h|^{2}\right\rangle-(m-1)|\nabla h|^{2} .
\end{aligned}
$$

Moreover, choosing an orthonormal frame $\left\{e_{i}\right\}_{i}$ such that $e_{1}=\frac{\nabla h}{|\nabla h|}$, we have

$$
\begin{aligned}
\left|h_{i j}\right|^{2} & \geq\left|h_{11}\right|^{2}+\sum_{\alpha>1}\left|h_{\alpha \alpha}\right|^{2}+2 \sum_{\alpha>1}\left|h_{1 \alpha}\right|^{2} \\
& \geq\left|h_{11}\right|^{2}+2 \sum_{\alpha>1}\left|h_{1 \alpha}\right|^{2}+\frac{1}{m-1}\left|\sum_{\alpha>1} h_{\alpha \alpha}\right|^{2} \\
& =\left|h_{11}\right|^{2}+2 \sum_{\alpha>1}\left|h_{1 \alpha}\right|^{2}+\left.\frac{1}{m-1}|| \nabla h\right|^{2}+\left.h_{11}\right|^{2} \\
& \geq \frac{m}{m-1}\left(\left|h_{11}\right|^{2}+\sum_{\alpha>1}\left|h_{1 \alpha}\right|^{2}\right)+\frac{1}{m-1}|\nabla h|^{4}+\frac{2}{m-1} h_{11}|\nabla h|^{2} .
\end{aligned}
$$

On the other hand, notice that

$$
\begin{aligned}
\left\langle\nabla|\nabla h|^{2}, \nabla h\right\rangle & =2 h_{i j} h_{i} h_{j}=2 h_{11}|\nabla h|^{2} \text { and } \\
\left.\left.|\nabla| \nabla h\right|^{2}\right|^{2} & =4\left|h_{i j} h_{j}\right|^{2}=4 h_{1 i}^{2}|\nabla h|^{2},
\end{aligned}
$$

which imply

$$
\left|h_{i j}\right|^{2} \geq\left.\left.\frac{m}{4(m-1)}|\nabla h|^{-2}|\nabla| \nabla h\right|^{2}\right|^{2}+\frac{1}{m-1}|\nabla h|^{4}+\frac{1}{m-1}\left\langle\nabla|\nabla h|^{2}, \nabla h\right\rangle .
$$

Then using this in (5) we conclude that

$$
\begin{gathered}
\frac{1}{2} \Delta|\nabla h|^{2} \geq\left.\left.\frac{m}{4(m-1)}|\nabla h|^{-2}|\nabla| \nabla h\right|^{2}\right|^{2}+\frac{1}{m-1}|\nabla h|^{4} \\
-\frac{m-2}{m-1}\left\langle\nabla|\nabla h|^{2}, \nabla h\right\rangle-(m-1)|\nabla h|^{2} .
\end{gathered}
$$


By (6) we have

$$
\begin{gathered}
\frac{1}{2} \Delta G \geq \frac{m}{4(m-1)} \phi^{4} G^{-1}\left|\nabla\left(\phi^{-2} G\right)\right|^{2}+\frac{1}{m-1} \phi^{-2} G^{2} \\
-\frac{m-2}{m-1} \phi^{2}\left\langle\nabla\left(\phi^{-2} G\right), \nabla h\right\rangle-(m-1) G+\frac{1}{2} \phi^{-2}\left(\Delta \phi^{2}\right) G+\left\langle\nabla \phi^{2}, \nabla\left(\phi^{-2} G\right)\right\rangle .
\end{gathered}
$$

At the maximum point $x_{0}$ it follows from (4) that

$$
\begin{aligned}
0 \geq & \frac{1}{m-1} G^{2}-(m-1) \phi^{2} G+\frac{m}{m-1}|\nabla \phi|^{2} G+\frac{2(m-2)}{m-1} \phi\langle\nabla \phi, \nabla h\rangle G \\
& +\frac{1}{2}\left(\Delta \phi^{2}\right) G-4|\nabla \phi|^{2} G .
\end{aligned}
$$

Since $\phi\langle\nabla \phi, \nabla h\rangle \geq-|\nabla \phi| G^{\frac{1}{2}}$ we infer from the above inequality that

$$
\begin{aligned}
0 \geq & \frac{1}{2}(m-1)\left(\Delta \phi^{2}\right) G-(3 m-4)|\nabla \phi|^{2} G-(m-1)^{2} \phi^{2} G \\
& -2(m-2)|\nabla \phi| G^{\frac{3}{2}}+G^{2} .
\end{aligned}
$$

This can be written as

$$
-(m-1) \phi \Delta \phi+(2 m-3)|\nabla \phi|^{2}+(m-1)^{2} \phi^{2}+2(m-2)|\nabla \phi| G^{\frac{1}{2}} \geq G .
$$

We point out that (7) is true for any cutoff $\phi$, but now we want to estimate the left-hand side of (7) from above using our choice of cutoff $\phi$. In existing arguments in the literature one would bound $(m-1)^{2} \phi^{2} \leq(m-1)^{2}$ and then deal with the terms involving $|\nabla \phi|$ and $\Delta \phi$. Our strategy is to make use of the fact that $\phi$ is small near the boundary. So, if $x_{0}$ is close to $\partial B_{p}(R)$, then it will be easy to see that $G\left(x_{0}\right) \leq(m-1)^{2}$. On the other hand, if that is not true, then $|\nabla \phi|$ and $\Delta \phi$ will be very small for our choice of $\phi$, because $\phi$ decays very slowly until the boundary.

First, observe that using a standard local gradient estimate (1), we can estimate

$$
\sup _{B_{p}(R)}|\nabla h| \leq(m-1)+\frac{c_{1}}{R},
$$

where $c_{1}$ is a constant depending only on $m$. Therefore, choosing $R_{0}(m)$ sufficiently large, we can guarantee that $(m-1)+\frac{c_{1}}{R} \leq m$. Consequently,

$$
G^{\frac{1}{2}}\left(x_{0}\right)=|\nabla h|\left(x_{0}\right) \phi\left(x_{0}\right) \leq \sup _{B_{p}(R)}|\nabla h| \leq m .
$$

Next, let us consider the function $\theta:[0, R] \rightarrow \mathbb{R}$,

$$
\theta(r)=\frac{R^{2}}{r^{2}} \exp \alpha\left(R-\frac{R^{2}}{r}\right) .
$$

Then we see that

$$
\theta^{\prime}(r)=\left(\alpha \frac{R^{4}}{r^{4}}-2 \frac{R^{2}}{r^{3}}\right) \exp \alpha\left(R-\frac{R^{2}}{r}\right),
$$

so by assuming $R_{0}>\frac{2}{\alpha}$ it follows that $\theta$ is increasing on $[0, R]$. Consequently,

$$
\theta(r) \leq \theta(R)=1 .
$$

By (3), we have thus proved that

$$
|\nabla \phi| \leq \alpha
$$


Furthermore, the Laplacian comparison theorem states that

$$
\Delta r\left(x_{0}\right) \leq(m-1)\left(1+\frac{1}{r\left(x_{0}\right)}\right)
$$

so that we find

$$
\begin{aligned}
-\Delta \phi & =-\phi^{\prime} \Delta r-\phi^{\prime \prime} \\
& \leq\left(\alpha(m-1) \frac{R^{2}}{r^{2}}\left(1+\frac{1}{r}\right)+\left(-2 \alpha \frac{R^{2}}{r^{3}}+\alpha^{2} \frac{R^{4}}{r^{4}}\right)\right) \exp \alpha\left(R-\frac{R^{2}}{r}\right) \\
& =\alpha\left((m-1) \frac{R^{2}}{r^{2}}+(m-3) \frac{R^{2}}{r^{3}}+\alpha \frac{R^{4}}{r^{4}}\right) \exp \alpha\left(R-\frac{R^{2}}{r}\right) .
\end{aligned}
$$

Consequently, since $0 \leq \phi \leq 1$ we get

$$
-\phi \Delta \phi \leq \alpha\left((m-1) \frac{R^{2}}{r^{2}}+(m-3) \frac{R^{2}}{r^{3}}+\alpha \frac{R^{4}}{r^{4}}\right) \exp \alpha\left(R-\frac{R^{2}}{r}\right) .
$$

Plugging all these into (77) and using (3) and (8) it follows that at $x_{0}$,

$$
\begin{aligned}
G \leq & (m-1)^{2} \phi-(m-1) \phi \Delta \phi+(2 m-3) \alpha|\nabla \phi|+2 m(m-2)|\nabla \phi| \\
\leq & (m-1)^{2}\left(1-\exp \alpha\left(R-\frac{R^{2}}{r}\right)\right) \\
& +\left\{\alpha\left(2 m(m-2)+(2 m-3) \alpha+(m-1)^{2}\right) \frac{R^{2}}{r^{2}}\right. \\
& \left.\quad+\alpha(m-1)(m-3) \frac{R^{2}}{r^{3}}+(m-1) \alpha^{2} \frac{R^{4}}{r^{4}}\right\} \exp \alpha\left(R-\frac{R^{2}}{r}\right) .
\end{aligned}
$$

We can simplify this using that

$$
\frac{R^{2}}{r^{2}} \leq \frac{R^{4}}{r^{4}}, \quad \frac{R^{2}}{r^{3}} \leq \frac{R^{4}}{r^{4}}
$$

and furthermore that for $\alpha \leq 2 / 3$,

$$
2 m(m-2)+(3 m-4) \alpha+(m-1)^{2}+(m-1)(m-3) \leq 4(m-1)^{2} .
$$

It follows that at $x_{0}$ we have

$$
G \leq(m-1)^{2}+(m-1)^{2}\left(4 \alpha \frac{R^{4}}{r^{4}}-1\right) \exp \alpha\left(R-\frac{R^{2}}{r}\right) .
$$

Let us now denote $u:[0, R] \rightarrow \mathbb{R}$

$$
u(r)=\left(4 \alpha \frac{R^{4}}{r^{4}}-1\right) \exp \alpha\left(R-\frac{R^{2}}{r}\right) .
$$

We want to find the maximum value of $u$ on $[0, R]$, which by (9) will imply the desired estimate for $|\nabla h|(p)$. We have the following two cases:

Case 1: $r \geq(4 \alpha)^{\frac{1}{4}} R$

Then it is easy to see that $u(r) \leq 0$.

Case 2: $r<(4 \alpha)^{\frac{1}{4}} R$

In this case it follows trivially that

$$
u(r) \leq 4 \alpha \frac{R^{4}}{r^{4}} \exp \alpha\left(R-\frac{R^{2}}{r}\right) .
$$


It is then easy to see that the function $w:[0, R] \rightarrow \mathbb{R}$,

$$
w(r)=\frac{R^{4}}{r^{4}} \exp \alpha\left(R-\frac{R^{2}}{r}\right),
$$

is increasing on $[0, R]$ if we assume $R_{0} \geq \frac{4}{\alpha}$.

Indeed,

$$
w^{\prime}(r)=\left(\alpha \frac{R^{6}}{r^{6}}-4 \frac{R^{4}}{r^{5}}\right) \exp \alpha\left(R-\frac{R^{2}}{r}\right) \geq 0
$$

if

$$
r \leq \frac{\alpha}{4} R^{2}
$$

But this is clearly true using that $R \geq R_{0} \geq \frac{4}{\alpha}$ and that $r \leq R$.

We have proved that $w$ is increasing on $[0, R]$; therefore for any $r<(4 \alpha)^{1 / 4} R$ we get

$$
\begin{aligned}
u(r) & \leq 4 \alpha w(r) \leq 4 \alpha w\left((4 \alpha)^{1 / 4} R\right) \\
& =\exp \left(-\alpha\left((4 \alpha)^{-1 / 4}-1\right) R\right) \\
& =\exp \left(-C_{2} R\right) .
\end{aligned}
$$

Note that $\alpha$ can be chosen to be a number not depending on $m$, and then $C_{2}$ is independent of $m$, too. For example, we can take $\alpha=2^{-6}$ and then $C_{2}=2^{-6}$.

Summing up, based on Case 1 and Case 2, it follows that for $R \geq R_{0}$,

$$
\begin{aligned}
|\nabla \log f|^{2}(p) & \leq G\left(x_{0}\right) \\
& \leq(m-1)^{2}+(m-1)^{2} \exp \left(-C_{2} R\right),
\end{aligned}
$$

which is what we claimed.

This proves the local gradient estimate, when the distance function from $p$ is smooth at $x_{0}$, the maximum point of $G$. As mentioned in the beginning, if this is not the case, we consider $\gamma(t)$, the minimizing geodesic from $p$ to $x_{0}$. Certainly, we may assume that $x_{0} \neq p$, since otherwise the estimates are trivial based on the definition of $\phi$.

Then take $q=\gamma(\varepsilon)$ for $\varepsilon$ small and define

$$
\psi(x):=\phi(d(q, x)+\varepsilon) .
$$

Since $x$ is in the cut locus of $p$, then it is not in the cut locus of $q$, so $\psi$ is smooth at $x_{0}$. Moreover, we have $d(q, x)+\varepsilon \geq r(x)$ for any $x \in M$ and $d\left(q, x_{0}\right)+\varepsilon=r\left(x_{0}\right)$. Therefore, using that $\phi$ is decreasing on $\mathbb{R}$ it follows that $\psi(x) \leq \phi(x)$ for any $x \in M$ and $\psi\left(x_{0}\right)=\phi\left(x_{0}\right)$.

This means that $x_{0}$ is the maximum point of $\widetilde{G}:=\psi^{2}|\nabla h|^{2}$, which now is smooth at $x_{0}$. Performing all the above computations and letting $\varepsilon \rightarrow 0$ it is not difficult to see that we still obtain (9) at $x_{0}$. The rest of the argument is the same.

This proves (2), which as explained in the beginning of the proof also proves the theorem.

As a consequence, we get the following sharp lower bound for the Green's function, by integrating the estimate in Theorem 1 along minimizing geodesics. We call $M$ nonparabolic if it admits a positive symmetric Green's function. 
Corollary 1. Let $M$ be a complete noncompact Riemannian manifold of dimension $m$ with Ric $\geq-(m-1)$ on $M$. If $M$ is nonparabolic, then there exists a constant $C$ depending only on $m$ such that

$$
\sup _{x \in \partial B_{p}(R)} G(p, x) \geq C \inf _{y \in \partial B_{p}(1)} G(p, y) \cdot e^{-(m-1) R},
$$

where $G(p, x)$ is the positive symmetric Green's function with a pole at $p \in M$.

On $\mathbb{H}^{n}$ let us now take Green's function $f(x):=G(q, x)$ with a pole at $q$. It is known that we have the following formula:

$$
f(x)=\int_{d(q, x)}^{\infty} \frac{d t}{A(t)}
$$

where $A(t)$ is the area of $\partial B_{q}(t)$. Then it can be checked that for $p$ such that $d(p, q)=R \geq 1$,

$$
(n-1)+C^{-1} e^{-2 R} \leq|\nabla \log f|(p) \leq(n-1)+C e^{-2 R} .
$$

This shows that the exponential-type decay in our theorem is sharp. Also, (10) shows that the decay estimate in the corollary is sharp.

\section{REFERENCES}

[1] E. Calabi, An extension of E. Hopf's maximum principle with an application to Riemannian geometry, Duke J. Math. 25 (1958), 45-56. MR0092069 (19:1056e)

[2] S.Y. Cheng and S.T. Yau, Differential equations on Riemannian manifolds and their geometric applications, Comm. Pure Appl. Math. 28 (1975), 333-354. MR0385749 (52:6608)

[3] P. Li, Harmonic functions and applications to complete manifolds, Instituto de Matemática Pura e Aplicada, Rio de Janeiro, 2006. MR.2369440 (2009a:53056)

[4] P. Li and J. Wang, Complete manifolds with positive spectrum, II, J. Differential Geom. 62 (2002), 143-162. MR1987380 (2004d:58045)

[5] R. Schoen and S. T. Yau, Lectures on Differential Geometry. International Press, Boston, 1994. MR1333601 (97d:53001)

[6] X. Wang, Harmonic functions, entropy, and a characterization of the hyperbolic space. J. Geom. Anal. 18 (2008), 272-284. MR2365675 (2009b:53062)

[7] S.T. Yau, Harmonic functions on complete Riemannian manifolds, Comm. Pure Appl. Math. 28 (1975), 201-228. MR0431040(55:4042)

Department of Mathematics, Columbia University, New York, New York 10027

E-mail address: omuntean@math.columbia.edu 